\title{
Tendinopatias do quadril: Uma atualização de conceitos e abordagens
}

\section{Hip Tendinopathies: An Update of Concepts and Approaches}

\author{
Vanessa Ribeiro de Resende ${ }^{1}$ (1) Yuri Rafael dos Santos Franco ${ }^{2}$ \\ ${ }^{1}$ Grupo de Ortopedia e Traumatologia do Esporte, Santa Casa de \\ Misericórdia de São Paulo, São Paulo, SP, Brasil \\ 2 Professor Doutor da Universidade de Guarulhos, São Paulo, SP, Brasil \\ Endereço para correspondência Vanessa Ribeiro de Resende, COTE - \\ Clínica de Ortopedia e Traumatologia do Esporte, Rua Martinico \\ Prado, 26, cj 192, Higienópolis, São Paulo, SP, 01224-010, Brasil \\ (e-mail: vanessares@yahoo.com.br). \\ Rev Bras Ortop 2022;57(3):369-374.
}

\section{Resumo \\ Palavras-chave \\ - tendinopatia \\ - extremidade inferior \\ - quadril \\ - lesões do quadril}

A presente atualização foi embasada nas novas evidências científicas das principais tendinopatias relacionadas ao quadril. Foram abordadas temáticas que envolvem os princípios do aparecimento das tendinopatias através, principalmente, do princípio da capacidade versus demanda e os aspectos biomecânicos envolvidos no seu aparecimento, suas principais características e apresentações clínicas. Associadas a isso, foram expostas as atualizações voltadas ao tratamento, com a terapia por exercício sendo o foco do tratamento conservador e as abordagens cirúrgicas necessárias para o controle ou resolução desses casos.

The present update was based on new scientific evidence of major hip-related tendinopathies. Themes were addressed that involve the principles of the onset of tendinopathies through, mainly, the principle of capacity versus demand and the biomechanical aspects involved in its onset, its main characteristics, and clinical presentations. Associated with this, treatment-related updates were presented, with exercise therapy being the focus of conservative treatment and surgical approaches necessary for the control or resolution of these cases.

\section{Introdução}

Tendinopatia é o termo mais correto e atual para se falar no contexto de doença do tendão. Este termo é conhecido como guarda-chuva, pois nele se aplicam diversas outras condições como a tendinite ou tendinopatia reativa e as tendinoses, desde o âmbito microscópico até o macroscópico. ${ }^{1}$

A fisiopatologia desta condição clínica ainda é incerta, pois diversas condições podem estar envolvidas, desde condições intrínsecas e sistêmicas como diabetes e obesidade e condições extrínsecas relacionadas, principalmente por sobrecargas. Neste ponto, aplica-se muito a teoria da capacidade e demanda, na qual muitas vezes a demanda excede a capacidade do sistema de suportar a carga imposta, seja ela no treinamento ou até mesmo no cotidiano do paciente. ${ }^{2}$

Por ser um tecido com especificidade bem explícita, onde ele é capaz de transmitir força do musculo ao osso, recebido

04 de Abril de 2021

aceito após revisão

14 de Maio de 2021

Publicado on-line

de Janeiro 24, 2022
DOI https://doi.org/

10.1055/s-0041-1736527.

ISSN 0102-3616. (c) 2022. Sociedade Brasileira de Ortopedia e Traumatologia. All rights reserved.

This is an open access article published by Thieme under the terms of the Creative Commons Attribution-NonDerivative-NonCommercial-License, permitting copying and reproduction so long as the original work is given appropriate credit. Contents may not be used for commercial purposes, or adapted, remixed, transformed or built upon. (https://creativecommons.org/ licenses/by-nc-nd/4.0/)

Thieme Revinter Publicações Ltda., Rua do Matoso 170, Rio de Janeiro, RJ, CEP 20270-135, Brazil 
principalmente, seu aspecto anaeróbico lhe dá a capacidade de resistir por mais tempo a sobrecargas; porém, quando este processo é quebrado, ou seja, existe alguma lesão ao tendão, o processo de cicatrização fica dificultado. ${ }^{3}$

Na maioria dos tratamentos vistos hoje na literatura, as opções de tratamento estão embasadas em iniciar com um tratamento conservador baseado em exercícios por pelo menos 12 meses. Caso haja falha nesta abordagem, o tratamento cirúrgico pode ser evidenciado. De uma maneira geral o uso de infiltrações de corticosteroides é uma conduta não indicada, mesmo trazendo efeitos positivos no curto prazo; porém, seu efeito a longo prazo não é benéfico, podendo evoluir até com rupturas do tendão. Esta mesma recomendação se aplica a terapias como repouso e imobilização. ${ }^{4}$

\section{Implicações Biomecânicas do Quadril}

A articulação do quadril é uma articulação sinovial do tipo esferoide. Sendo assim, é uma articulação que tem em sua anatomia uma característica estável por se tratar de uma arquitetura bola e soquete.

Sobre a biomecânica desta articulação, é válido pensar em suas amplitudes e que suas ações acontecem nas duas cadeias cinéticas, sendo a cadeia cinética fechada a mais funcional. Vale salientar o valgo dinâmico, que tem em sua biomecânica uma adução e rotação interna em cadeia cinética fechada, uma situação na qual as forças de cisalhamento são bem evidentes, o que exige da musculatura uma necessidade maior de estabilização e com os músculos se apresentando sobrecarregados. Neste exemplo, vale salientar que a força dos abdutores precisa estar equiparada à dos adutores para que se tenha este controle no plano frontal de maneira mais efetiva. 0 mesmo acontece com os rotadores laterais, que não apresentam antagonistas primários, que precisam estar com força igual ou superior a três vezes o peso corporal, segundo a balança de Pauwells. ${ }^{5}$

Um ponto interessante a ser falado sobre a articulação do quadril é que, por este ter o acetábulo em sua composição e essa estrutura ser composta pelos três ossos da pelve, qualquer desarranjo que venha a interferir no acetábulo acarreta uma sobrecarga na sínfise púbica e na articulação sacroilíaca, o que leva a compensações em toda a estrutura funcional no complexo da pelve. ${ }^{6}$

Estes conceitos são muito reducionistas nos contextos atuais da biomecânica, pois se sabe que as alterações advindas do quadril não são a única fonte de compensações para o valgo dinâmico. Atualmente, temos alterações de origem mais distal, como por exemplo a limitação de dorsiflexão que, devido a toda cadeia lesional, pode interferir a nível do quadril gerando as compensações acima citadas e, assim, as possíveis tendinopatias na região. Vale salientar que esta limitação de dorsiflexão tem diversas origens, porém as entorses do tornozelo geram um importante referencial que pode ser avaliado pelo teste de Lunge. ${ }^{7}$

\section{Sindrome do Trocanter Maior}

Atualmente, a nomenclatura para sintomas que aparecem na lateral do quadril é síndrome da dor trocantérica maior (GTPS, na sigla em inglês) e inclui pacientes com sintomas de dor peritrocantérica, tendinopatia do glúteo médio/mínimo, as bursites trocantéricas e a síndrome do ressalto externo. ${ }^{8}$

Sendo assim, podem existir condições nas quais a tendinopatia não esteja presente; porém, o que observamos é que o tratamento é muito semelhante para as diferentes patologias locais. ${ }^{9}$

\section{Tendinopatia Glútea}

Se a principal característica está voltada para falha da balança entre capacidade e demanda, o aumento da demanda ocorrerá devido a um excesso de treinamento ou até mesmo pelo destreinamento para as atividades cotidianas. 0 perfil de pacientes do sexo feminino com maior idade mostra essa ação do destreinamento. No caso de populações mais jovens, a prevalência está em mulheres que praticam alguma atividade física de impacto que envolva corrida. Este fato pode ser explicado por uma visão mais biomecânica sobre a vertente da falha da balança de Pauwells, onde existe uma desvantagem natural das mulheres na anatomia (pelve mais larga) e uma diminuição da força e da velocidade de contração, fatores que podem interferir negativamente para que exista um desnivelamento da pelve (adução do quadril em cadeia cinética fechada) e, com isso, um aumento na demanda de ação dos abdutores do quadril e rotadores laterais do quadril. ${ }^{5}$

Com isso, bem marcada pela literatura, a principal abordagem se dá através de terapias por exercícios, com foco em reestruturar a força dos abdutores do quadril e rotadores laterais do quadril (- Figura 1). A associação de crioterapia

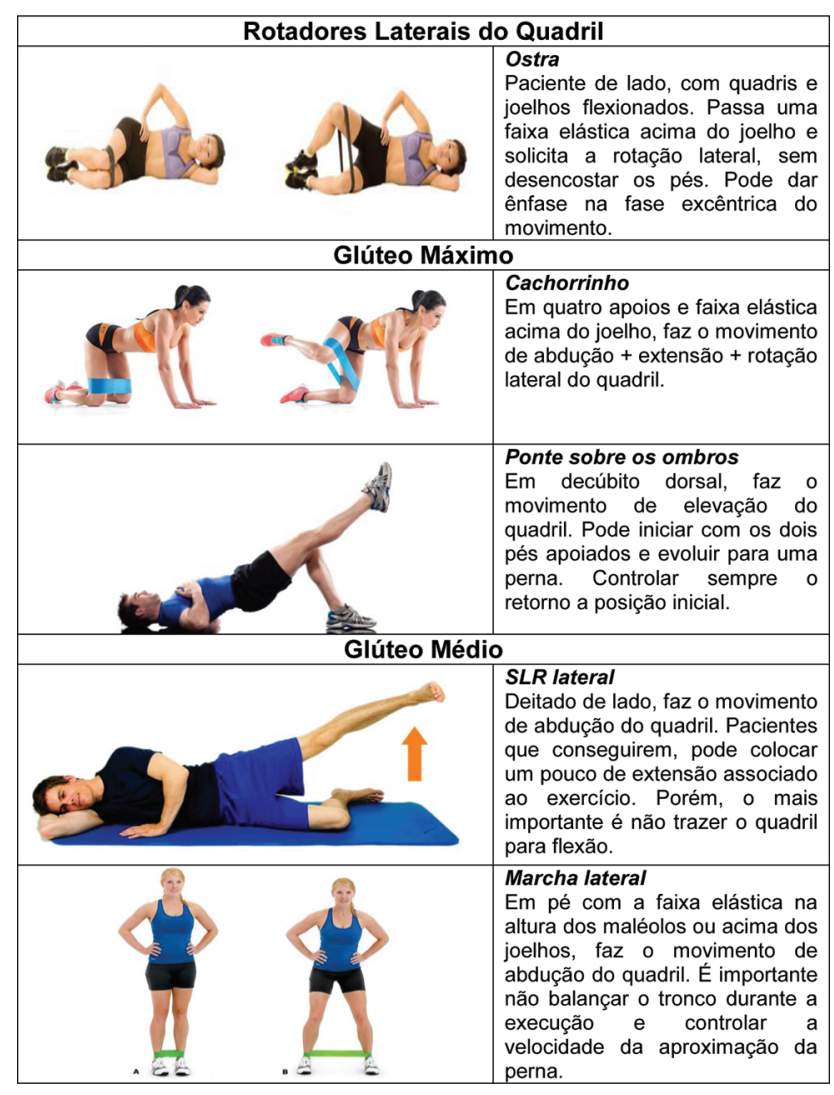

Fig. 1 Exercícios básicos iniciais de orientação aos pacientes para fortalecimento global do quadril. 
local e uma diminuição, e não repouso total, na demanda (treino e/ou atividades rotineiras) são aliados para uma boa evolução dos casos. Alguns autores defendem o alongamento da banda iliotibial (ITB) e injeções de cortisona no tratamento não operatório. ${ }^{8}$

A terapia por ondas de choque de baixa energia pode ser uma opção de tratamento e a justificativa para seu uso na tendinopatia é que ela pode causar analgesia de hiperestimulação através da liberação de citocinas nos tecidos afetados. Estes, então, interrompem a ativação serotonérgica que exercem controle inibitório descendente sobre a dor. ${ }^{10}$ Quando o diagnóstico é estabelecido por meio de exame físico meticuloso e ressonância magnética (RM), ela parece ser uma opção de tratamento eficaz para o alívio da dor refratária crônica. No entanto, seu efeito a longo prazo parece diminuir com o tempo. ${ }^{11}$

Técnicas de reparo aberto e artroscópico foram descritas na literatura recente, demonstrando excelentes resultados relatados pelos pacientes. ${ }^{12}$ Quando a cirurgia é indicada devido ao fracasso de estratégias não operatórias, a zetaplastia aberta no nível do trocanter maior tem sido o procedimento tradicional. A liberação endoscópica do ITB e a bursectomia ao nível do trocanter maior evoluíram nas últimas décadas e se estabeleceram como um método alternativo de cirurgia. ${ }^{8}$

\section{Síndrome do Glúteo Médio}

Definida como uma síndrome de dor miofascial (dor acompanhada pela confirmação de pontos-gatilho de dor em músculos específicos) decorrente do glúteo médio, esta é uma das principais causas de dor nas costas ou nas pernas e é semelhante à síndrome da dor trocantérica maior, que também se manifesta com dor nas costas ou nas pernas, porém comumente está relacionada à doença degenerativa lombar, osteoartrite do quadril, osteoartrite do joelho e à síndrome da cirurgia fracassada nas costas. Ela é tratada por fisioterapia, terapia de liberação manual do ponto de gatilho ou injeção de bloqueio de ponto de gatilho e, em casos difíceis, por descompressão cirúrgica do glúteo médio ou do nervo ciático. ${ }^{13}$

\section{Sindrome do Quadril Estalante}

A síndrome do ressalto do quadril, também conhecida como coxa saltans (ou quadril do dançarino), é uma condição clínica caracterizada por uma sensação de estalo audível ou palpável que é ouvida durante o movimento da articulação do quadril. O ressalto do quadril tem múltiplas etiologias e é classificado com base na estrutura anatômica que é a causa/fonte da sensação de ressalto.

O ressalto externo do quadril é mais comumente atribuído ao movimento da banda iliotibial sobre o trocanter maior da cabeça femoral durante os movimentos do quadril em flexão, extensão e rotação externa ou interna. Outras causas incluem o tendão do isquiotibial proximal rolando sobre a tuberosidade isquiática, seja a fáscia lata ou o aspecto anterior do glúteo máximo, rolando sobre o trocanter maior e o tendão do psoas rolando sobre as fibras mediais do músculo ilíaco. Uma combinação de defeitos também é possível; por exem- plo, espessamento da banda iliotibial posterior e do glúteo máximo anterior, que se encaixam no trocânter maior ao mesmo tempo.

Quando a dor não está presente, o tratamento não se justifica. Quando a dor está presente no estalido, o tratamento é conservador e consiste em repouso, alongamento, injeções de esteroides, medicamentos anti-inflamatórios orais, fisioterapia e modificação da atividade. Na maioria das vezes, os pacientes sentem alívio com estas medidas. ${ }^{14}$

0 ressalto interno do quadril ocorre mais comumente quando o tendão do iliopsoas se encaixa nas proeminências ósseas subjacentes, como a eminência iliopectinal ou a face anterior da cabeça do fêmur. Outras causas incluem cistos paralabrais e bifurcação parcial ou completa do tendão iliopsoas. A sensação de estalo pode imitar de perto a patologia intra-articular, uma vez que ambos se originam na área anterior do quadril. $\mathrm{O}$ exame físico e as imagens de perto podem diferenciar os dois. Deve-se notar, entretanto, que em $50 \%$ dos casos de ressalto interno do quadril, uma patologia intra-articular adicional do quadril é identificada. ${ }^{15}$

Se a dor persistir apesar destas medidas conservadoras, a intervenção cirúrgica pode ser considerada. Para a síndrome de ressalto externo do quadril, o afrouxamento da banda iliotibial geralmente é o objetivo e pode ser realizado com procedimentos abertos ou artroscópicos. O tendão iliotibial é alongado ou completamente liberado usando vários procedimentos, incluindo alongamento formal em Z, liberação em forma de cruz, liberação em forma de $\mathrm{Z}$ ou liberação do glúteo máximo. Fraqueza na abdução pode ser uma complicação se a liberação for excessiva ou se houver danos à área circundante. $^{14}$

Para a síndrome do ressalto interno do quadril, procedimentos abertos ou artroscópicos também estão disponíveis para alongar ou liberar o tendão iliopsoas. Métodos artroscópicos são preferidos para evitar complicações da cirurgia aberta. $O$ efeito adverso mais comum da liberação do iliopsoas é a fraqueza dos flexores do quadril, que pode ocorrer se houver liberação excessiva ou danos na área circundante. As cirurgias corretivas para o quadril com ressalto interno ou externo podem resultar em outras complicações, incluindo infecção, ossificação heterotópica, atrofia muscular, sintomas contínuos ou danos aos nervos. ${ }^{2}$

\section{Sindrome do Piriforme}

A síndrome do piriforme (SP) é uma doença incomum e controversa que se presume ser uma neuropatia de compressão do nervo ciático ao nível do músculo piriforme (PM). È uma irritação do músculo piriforme, um pequeno músculo abaixo do glúteo máximo. Irritação pode ocorrer se permanecemos por longos períodos sentados ou de pé, ou fazemos repetição de certos movimentos. Quando o músculo piriforme apresenta-se endurecido e com espasmo, ele pode comprimir o nervo ciático. A irritação, chamada SP, pode causar dor e parestesia (dormência e formigamento) no glúteo mínimo, da parte posterior da perna até o pé. Os sintomas são confundidos com doenças discais da coluna. Uma maneira de afirmar que é SP é dobrar seus joelhos junto ao corpo, com seus pés no ar. Gentilmente vá afastando os pés 
um para cada lado. Se houver dor nos glúteos quando mover o pé, suspeite de SP. O tratamento tem se concentrado em alongamento, fisioterapia, injeções locais, incluindo toxina botulínica e manejo cirúrgico. ${ }^{16}$

\section{Reto Femoral Proximal}

0 reto femoral tem sua característica anatômica e biomecânica por ser um músculo biarticulado que atua juntamente com o quadríceps na extensão do joelho e isoladamente na flexão do quadril. Seu papel, além de funcional, tem um aspecto de estabilidade da porção anterior desta articulação. A sua ação de estabilização é similar à do Iliopsoas, que auxilia na flexão do quadril. ${ }^{17} \mathrm{~A}$ etiologia da sua sobrecarga ainda é imprecisa, mas sabe-se que o aumento da sua demanda ocorre em esportes de velocidade, principalmente na fase de desaceleração brusca e no futebol nos chutes longos.

O principal público afetado está nas extremidades das faixas de idade, sendo crianças em seu processo de iniciação no esporte ou pessoas com mais idade que necessitam de uma maior demanda. Muitas vezes, pode ocorrer avulsão desta estrutura junto à espinha ilíaca anteroinferior. Em pacientes mais jovens, o acometimento está diretamente relacionado ao aumento das demandas, especificamente dos esportes como futebol, associado a lesões prévias de quadríceps e com o biotipo de pessoas mais baixas e com sobrepeso. $^{18}$

Sobre o tratamento, para os casos de tendinopatia, o foco deve estar em equilibrar as condições de demanda com aumento da força dessas estruturas, lembrando que o fortalecimento específico da região é interessante, ou seja, movimentos voltados para flexão do quadril. Outra consideração interessante está na utilização de exercícios voltados para as características mais excêntricas, visando a especificidade do gesto do paciente, assim como a demanda metabólica deste músculo. ${ }^{19}$

\section{Adutores do Quadril}

Este conjunto de músculos tem um papel importante para a articulação do quadril, pois tem em sua ação principal aduzir o fêmur; porém, suas ações secundárias estão ligadas à flexão e à extensão do quadril, ou seja, mesmo sem estar fazendo sua ação principal, sua ativação é quase que contínua. Esta condição aumenta a sua demanda, podendo estar ligada a isso a presença de sua tendinopatia. ${ }^{20}$

Esta condição é uma das principais relacionadas a dor na virilha, sendo o tendão do adutor longo o mais acometido. Dor crônica na virilha no atleta pode ser um problema difícil de controlar. $\mathrm{O}$ achado mais reprodutível para tendinopatia adutora longa é sensibilidade ao longo do tendão com abdução passiva e adução resistida do quadril em extensão. ${ }^{20}$

Existem algumas condições que podem estar ligadas ao aparecimento da tendinopatia dos adutores, dentre elas a síndrome do impacto femoroacetabular e a pubalgia; esta última ainda sem um consenso de causa e consequência, pois muito se pensou anteriormente somente o desarranjo no plano frontal da sínfise púbica, teorizando que existia envolvimento somente do reto abdominal e adutores que estão ligados diretamente na região púbica. ${ }^{21}$

Atualmente, acredita-se em movimento no plano sagital, também, evidenciando a importância entre flexores e extensores do quadril. Acredita-se que existe um desbalanço entre estes dois grupamentos e, como os adutores são sinergistas destes movimentos, sua ativação é aumentada, gerando assim maior tensão e sobrecarga. ${ }^{20}$ Com esta visão mais global da pubalgia, o tratamento deve ser focado não somente no balanço dos adutores, mas sim na região glútea e de isquiotibial, por estas terem sua ação na extensão e no iliopsoas e reto femoral, e por se tratar do grupamento flexor do quadril; assim, os adutores entrariam apenas como uma lapidação, pois muitas vezes eles não estão fracos e sim sobrecarregados. $^{21}$

A RM e a injeção de anestésico na junção músculo-tendão proximal podem ser úteis para confirmar o diagnóstico. ${ }^{20}$

A intervenção para esta tendinopatia está voltada para o controle de sintomas, principalmente através de exercícios específicos para a região e para que se consiga equilibrar a demanda e a capacidade para as atividades do cotidiano ou esportivas. ${ }^{22} \mathrm{O}$ tratamento não operatório pode consistir em suporte de peso protegido, aplicação de gelo, ultrassonografia, estimulação elétrica e alongamento suave com fortalecimento progressivo. ${ }^{5}$

No entanto, o tratamento não operatório nem sempre é bem-sucedido para tendinopatia crônica. Nestes casos, o tratamento cirúrgico pode ser bastante eficaz, através da tenotomia adutora, a qual pode ser uma ferramenta útil para o tratamento da dor recalcitrante na virilha atribuível ao adutor longo. ${ }^{14}$

TENDINOPATIA PROXIMAL DOS ISQUIOTIBIAIS (IQT) (Tendinopatia alta dos IQT, Síndrome da intersecção isquiática, Entesopatia dos IQT ou tendinopatia da origem dos IQT)

Lesões nos isquiotibiais (IQTs) são uma das lesões mais comuns sofridas por atletas. Estas lesões costumam ocorrer em velocistas ou corredores de média a longa distância, e variam de entorses e rupturas agudas a lesões crônicodegenerativas que ocorrem como resultado de pequenas cargas repetitivas e trauma na origem dos tendões dos IQTs da tuberosidade isquiática. ${ }^{23}$

O complexo dos IQTs é composto por três músculos: semimembranoso, semitendíneo e bíceps femoral. A cabeça longa do bíceps femoral e os músculos semitendíneos formam o tendão conjunto que se insere no aspecto posteromedial da tuberosidade isquiática. ${ }^{23}$

A tendinopatia proximal dos IQTs é uma doença crônicodegenerativa associada a morbidade progressiva e declínio funcional. Há uma incidência crescente do processo da doença, mas o diagnóstico é comumente retardado, pois os pacientes apresentam sintomas vagos e indolentes, muitas vezes sem uma lesão precipitante específica. ${ }^{23}$

As principais funções dos músculos IQTs são extensão do quadril e flexão do joelho com inervação primária do nervo ciático (tibial). Todos os três músculos recebem seu suprimento sanguíneo de ramos das artérias femoral e glútea inferior. Amostras de biópsia intraoperatória de pacientes 
submetidos à tenotomia por tendinopatia mostraram que os músculos IQTs podem ser afetados isoladamente ou como uma tríade complexa com vários graus de inflamação dentro de cada tendão proximal. $^{23}$

Fatores intrínsecos levam a anormalidades estruturais na sua origem proximal que predispõem o tendão ao risco aumentado de lesão e potencial de cura reduzido. Os tendões IQTs de pacientes mais velhos mostram uma capacidade inferior das células-tronco do tendão para estimular a clonogenicidade, indutibilidade adiposa e indutabilidade osteogênica. ${ }^{24} \mathrm{Em}$ mulheres na perimenopausa, os níveis reduzidos de estrogênio podem ter um impacto adverso na hemostasia e na cura do tendão, levando ao colapso progressivo e degenerativo do tendão. Outros fatores intrínsecos associados à tendinopatia do IQT proximal incluem predisposição genética (por exemplo, mutações em COL5A1 que codifica para colágeno tipo $\mathrm{V}$ ), anormalidades metabólicas (desequilíbrio do nível de lipídios, intolerância à glicose, resistência à insulina), alterações hormonais e agentes farmacológicos (por exemplo, antibióticos fluoroquinolonas). ${ }^{23}$

Fatores extrínsecos podem promover aumento da carga de trabalho e carga excêntrica na origem dos IQT proximais, e a compressão dos tendões nessas origens durante a flexão e adução do quadril pode exacerbar os sintomas. Aumento da flexão do quadril leva a maiores forças de cisalhamento entre a tuberosidade isquiática e os tendões dos IQTs, com maior deslocamento dos IQTs proximais. Erros de treinamento que aumentam o volume e a duração do treinamento de forma muito vigorosa ou introduzem exercícios como corrida de velocidade ou obstáculos podem desencadear a tendinopatia proximal. Estas atividades causam rápida contração e alongamento enquanto o quadril está em flexão, o que gera maiores cargas de tração e compressão na sua inserção. 0 posicionamento anormal do quadril em posturas de pilates e ioga podem provocar sintomas semelhantes. ${ }^{18}$

Pacientes frequentemente relatam aumento gradual da dor ou desconforto na região subglútea ou posterior da coxa. Esta dor é descrita como 'cãibra' ou 'aperto' na área das nádegas profundas e geralmente progride ao longo do tempo sem nenhum trauma ou lesão incitante específicos. A radiação para a fossa poplítea pode causar inibição da dor e fraqueza dos músculos IQTs e dificuldade de participação em atividades esportivas. Os sintomas podem ser exacerbados por carga excêntrica repetitiva ou flexão frontal prolongada do tronco, como durante exercícios de alongamento, corrida e se sentar por longos períodos. Em casos mais graves, a fibrose dos músculos IQTs proximais pode prender e comprimir o nervo ciático, causando dor aguda que se irradia da parte posterior da coxa até o pé. ${ }^{13}$

Alguns autores acreditam que os corticosteroides ajudam a limitar a inflamação crônica e, portanto, a reduzir a formação de cicatrizes e aderências no tendão. A administração de medicamentos sob orientação de ultrassom facilita a colocação precisa da injeção na bainha do tendão e evita a infiltração direta na substância do tendão. A resolução imediata dos sintomas com o anestésico local é uma ferramenta diagnóstica útil quanto à origem dos sintomas e indica que o medicamento foi administrado com precisão. ${ }^{25}$
Tratamento com ondas de choque pode ser utilizado, a justificativa para o seu uso na tendinopatia é que ele pode causar analgesia de hiperestimulação através da liberação de citocinas nos tecidos afetados. Estes, então, interrompem a ativação serotonérgica que exercem controle inibitório descendente sobre a dor. ${ }^{10}$

O plasma rico em plaquetas contém proteínas regulatórias, fatores de crescimento e plaquetas que inibem e modulam a ação das células pró-inflamatórias e facilitam a cicatrização tendínea mais rápida. No geral, os estudos usando plasma rico em plaquetas mostraram resultados promissores nos estágios iniciais, com melhora da dor e pontuação funcional no acompanhamento de curto a médio prazo. $^{23}$

No tratamento cirúrgico, uma opção é identificar e liberar no intraoperatório o provável tendão dos IQTs causador da tendinopatia por meio da visualização da unidade miotendinosa que apresentava cicatrizes, hipertrofia e fibrose. Rupturas agudas de isquiotibiais associadas à tendinopatia proximal de IQTs em atletas de alto nível podem se beneficiar da fixação com âncora de sutura do tendão avulsionado à tuberosidade isquiática ou reconstrução com aloenxerto do tendão de Aquiles para restaurar a função e ajudar no retorno às atividades esportivas. ${ }^{18}$

\section{Considerações Finais}

As tendinopatias do quadril são patologias comuns no dia-adia do ortopedista e, apesar do seu caráter multifatorial, a biomecânica tem papel fundamental nesta patologia, principalmente a relação carga-demanda, e por isso a importância da abordagem o mais precoce possível no fortalecimento e equilíbrio muscular destes pacientes. Ainda necessitamos de estudos para compreender melhor suas relações musculares nos diversos momentos do dia a dia, mas, na maioria dos casos, o tratamento conservador se faz eficiente, ficando o tratamento cirúrgico reservado a casos refratários.

\section{Suporte Financeiro}

Não houve suporte financeiro de fontes públicas, comerciais, ou sem fins lucrativos.

\section{Conflito de Interesses}

Os autores declaram não haver conflito de interesses.

\section{Referências}

1 Andres BM, Murrell GA. Treatment of tendinopathy: what works, what does not, and what is on the horizon. Clin Orthop Relat Res 2008;466(07):1539-1554

2 Khan K, Cook J. The painful nonruptured tendon: clinical aspects. Clin Sports Med 2003;22(04):711-725

3 Sharma P, Maffulli N. Tendon injury and tendinopathy: healing and repair. J Bone Joint Surg Am 2005;87(01):187-202

4 Challoumas D, Clifford C, Kirwan P, Millar NL. How does surgery compare to sham surgery or physiotherapy as a treatment for tendinopathy? A systematic review of randomised trials. BMJ Open Sport Exerc Med 2019;5(01):e000528

5 Neumann DA. Kinesiology of the hip: a focus on muscular actions. J Orthop Sports Phys Ther 2010;40(02):82-94 
6 Ou-Yang DC, York PJ, Kleck CJ, Patel VV. Diagnosis and Management of Sacroiliac Joint Dysfunction. J Bone Joint Surg Am 2017; 99(23):2027-2036

7 Lima YL, Ferreira VMLM, de Paula Lima PO, Bezerra MA, de Oliveira RR, Almeida GPL. The association of ankle dorsiflexion and dynamic knee valgus: A systematic review and meta-analysis. Phys Ther Sport 2018;29:61-69

8 Thomassen PJB, Basso T, Foss OA. Endoscopic Treatment of Greater Trochanteric Pain Syndrome - A Case Series of 11 Patients. J Orthop Case Rep 2019;9(01):6-10

9 Redmond JM, Chen AW, Domb BG. Greater Trochanteric Pain Syndrome. J Am Acad Orthop Surg 2016;24(04):231-240

10 Cacchio A, Rompe JD, Furia JP, Susi P, Santilli V, De Paulis F. Shockwave therapy for the treatment of chronic proximal hamstring tendinopathy in professional athletes. Am J Sports Med 2011;39(01):146-153

11 Seo KH, Lee JY, Yoon K, et al. Long-term outcome of low-energy extracorporeal shockwave therapy on gluteal tendinopathy documented by magnetic resonance imaging. PLoS One 2018;13(07): e0197460

12 LaPorte C, Vasaris M, Gossett L, Boykin R, Menge T. Gluteus medius tears of the hip: a comprehensive approach. Phys Sportsmed 2019;47(01):15-20

13 Kameda M, Tanimae H, Kihara A, Matsumoto F. Does low back pain or leg pain in gluteus medius syndrome contribute to lumbar degenerative disease and hip osteoarthritis and vice versa? A literature review. J Phys Ther Sci 2020;32(02):173-191

14 Badowski E. Snapping Hip Syndrome. Orthop Nurs 2018;37(06): 357-360

15 Musick SR, Varacallo M. Snapping Hip Syndrome. In: StatPearls. Treasure Island (FL): StatPearls Publishing; 2020
16 Probst D, Stout A, Hunt D. Piriformis Syndrome: A Narrative Review of the Anatomy, Diagnosis, and Treatment. PM R 2019; 11(Suppl 1):S54-S63

17 Pesquer L, Poussange N, Sonnery-Cottet B, et al. Imaging of rectus femoris proximal tendinopathies. Skeletal Radiol 2016;45(07): 889-897

18 Mendiguchia J, Alentorn-Geli E, Idoate F, Myer GD. Rectus femoris muscle injuries in football: a clinically relevant review of mechanisms of injury, risk factors and preventive strategies. $\mathrm{Br} J$ Sports Med 2013;47(06):359-366

19 Kubo Y, Watanabe K, Nakazato K, et al. The Effect of a Previous Strain Injury on Regional Neuromuscular Activation Within the Rectus Femoris. J Hum Kinet 2019;66:89-97

20 Gill TJ, Carroll KM, Makani A, Wall AJ, Dumont GD, Cohn RM. Surgical technique for treatment of recalcitrant adductor longus tendinopathy. Arthrosc Tech 2014;3(02):e293-e297

21 Hölmich P, Uhrskou P, Ulnits L, et al. Effectiveness of active physical training as treatment for long-standing adductor-related groin pain in athletes: randomised trial. Lancet 1999;353 (9151):439-443

22 Gilmore J. Groin pain in the soccer athlete: fact, fiction, and treatment. Clin Sports Med 1998;17(04):787-793

23 Pietrzak JR, Kayani B, Tahmassebi J, Haddad FS. Proximal hamstring tendinopathy: pathophysiology, diagnosis and treatment. $\mathrm{Br}$ J Hosp Med (Lond) 2018;79(07):389-394

24 Ruzzini L, Abbruzzese F, Rainer A, et al. Characterization of agerelated changes of tendon stem cells from adult human tendons. Knee Surg Sports Traumatol Arthrosc 2014;22(11):2856-2866

25 Zissen MH, Wallace G, Stevens KJ, Fredericson M, Beaulieu CF. High hamstring tendinopathy: MRI and ultrasound imaging and therapeutic efficacy of percutaneous corticosteroid injection. AJR Am J Roentgenol 2010;195(04):993-998 